\title{
BMJ Open Validation of a three-dimensional printed dry lab pancreaticojejunostomy model in surgical assessment: a cross- sectional study
}

\author{
Hao Yu (D) , ${ }^{1,2}$ Tunan Yu, ${ }^{3,4}$ Jiulong Wang, ${ }^{5}$ Fangqiang Wei, ${ }^{6}$ Haibo Gong, ${ }^{7}$ \\ Haiying Dong, ${ }^{8}$ Xinzhong He, ${ }^{9}$ Zhifei Wang, ${ }^{6}$ Jin Yang ${ }^{1,4}$
}

To cite: Yu H, Yu T, Wang J, et al. Validation of a threedimensional printed dry lab pancreaticojejunostomy model in surgical assessment: a crosssectional study. BMJ Open 2022;12:e052295. doi:10.1136/ bmjopen-2021-052295

- Prepublication history and additional supplemental material for this paper are available online. To view these files, please visit the journal online (http://dx.doi.org/10.1136/ bmjopen-2021-052295).

Received 13 April 2021 Accepted 06 January 2022
Check for updates

(C) Author(s) (or their employer(s)) 2022. Re-use permitted under CC BY-NC. No commercial re-use. See rights and permissions. Published by BMJ.

For numbered affiliations see end of article.

\section{Correspondence to}

Dr Zhifei Wang;

wangzhifei1973@zju.edu.cn and

Dr Jin Yang;

3319017@zju.edu.cn

\section{ABSTRACT}

Objectives Until now, there have been few tools to evaluate whether a surgeon was technically ready to perform a safe pancreaticojejunostomy (PJ). In the current study, we aimed to evaluate whether a three-dimensional model could mimic a real surgical situation and distinguish between surgeons of different levels of experiences. Design A three-dimensional PJ dry laboratory model was printed. Eight experienced pancreatic surgeons were tasked to evaluate the appearance and tactile sensation of the model. Proficiency was scored based on 15 surgeons with various levels of pancreatic experience performing a PJ on the three-dimensional model. Additionally, the time of manipulation and NASA Task Load Index (NASA-TLX) scores were recorded for each operation.

Setting Our study was conducted in multimedical centre in China.

Results Compared with real surgical situations, this model had similar appearance $(3.96 \pm 0.55$ out of five points) and tactile sensation $(3.85 \pm 0.46$ out of five points) according to the expert evaluation. Additionally, the chief surgeon group scored the best in proficiency (based on NASA-TLX scores and operative time), and there were statistical differences for performances among surgeons of various levels $(p<0.05)$.

Conclusion The three-dimensional PJ model could mimic a real surgical situation and can distinguish between surgeons of different levels of experiences.

\section{INTRODUCTION}

A pancreaticojejunostomy (PJ) is one of the most challenging procedures in general surgery. and a lack of proficiency and experience in doing this procedure may lead to postoperative pancreatic leakage, haemorrhage or even death. ${ }^{1-3}$ Advanced techniques, such as 3D printing, have been widely used in the field of surgery for the purpose of education and preoperative designing; however, there are few reports indicating that they could be used as a tool to evaluate surgical competency.

According to Szasz and colleagues, ${ }^{4}$ due to work hour restrictions, limitations of

\section{Strengths and limitations of this study}

- The three-dimensional pancreaticojejunostomy (PJ) model could mimic a real surgical situation, allowing it to be used as a portable teaching and learning tool.

- The model is easier to store; therefore, it can be used by students in the office or even at home.

- Although the current study used softer silicone material to simulate the pancreatic parenchyma, its structure was still slightly higher than that of the pancreatic tissue.

- The PJ model in the current study did not contain vessels such as the splenic artery, which would have allowed for the simulation of a more realistic situation.

- The characteristics of the pancreatic tissue (consistency, elasticity, etc) are highly different from one patient to another, which may influence both the technique and the results of pancreatoenteric anastomosis, but in the current study. only one type of silicon model was used.

operating room accessibility and increased litigation against physicians, the educational opportunities of surgeons have dramatically decreased. Based on this status quo, the Accreditation Council for Graduate Medical Education, ${ }^{5}$ the Royal College of Physicians and Surgeons of Canada ${ }^{6}$ and many others worldwide have developed training programmes to improve surgical skills.

Compared with traditional pancreaticoduodenal surgery training methods, there remains a lack of an effective physical model to help distinguish between pancreatic surgeons of different levels and to roughly assess whether pancreatic surgeons are prepared. As an emerging technology, 3D printing technology has been widely used in the medical field ${ }^{7-9}$ and has been broadly studied and reported in a book on the training and application of simulation models in robotic gynaecological surgery. ${ }^{10}$ 
Additionally, 3D-printed models are expected to be used in the future as one of the methods of pancreatic surgery training, reducing learning costs and helping young doctors improve surgical techniques. In the current study, experts in the field of pancreatic surgery were invited to evaluate the appearance of the model. We aimed to evaluate whether a three-dimensional model could mimic a real surgical situation and distinguish between surgeons of various levels of experience.

\section{Study design and setting}

The current revolutionary study invited eight surgical experts from multiple pancreatic surgery centres in China to conduct an anatomical evaluation of a 3D-printed model. All eight experts had performed more than 20 instances of pancreaticoduodenectomy within the prior year and four had performed more than 100 instances of pancreaticoduodenectomy in the prior year. Fifteen doctors from our pancreatic surgery centre were invited to participate in the model function evaluation.

\section{D-printed dry lab PJ model production}

The 3D-printed dry lab PJ model primarily contained the pancreas and small intestine and was printed using a dualhead silicone printer. S online supplemental appendix S1. First, the CT data were collected in a Digital Imaging and Communications in Medicine format, with $1 \mathrm{~mm}$ thick slices. The E3D digital medical modelling software V.17.06 (Central South E3D Digital Medical and Virtual Reality Research Center, China) was used for boundary segmentation and 3D reconstruction and the model structure were streamlined according to manual editing (figure 1). The open source slicing software Cura V.4.4.1 (Ulitmaker, USA) was used for slicing the 3D printing. The material was made of silicone specialised for 3D printing. The silicone material used for the pancreatic parenchyma was pink, with a tear strength of $4.8 \mathrm{~N} /$ $\mathrm{mm}$ and a tensile strength of $2 \mathrm{MPa}$. The silicone material used for the pancreatic duct was white, with a tear strength of $5.2 \mathrm{~N} / \mathrm{mm}$ and a tensile strength of $1.8 \mathrm{MPa}$. The silicone material used for the small intestine was red, with a tear strength of $5.2 \mathrm{~N} / \mathrm{mm}$ and a tensile strength of $1.8 \mathrm{MPa}$. The pancreas was the primary component of the PJ model and its stiffness was measured via ultrasound,

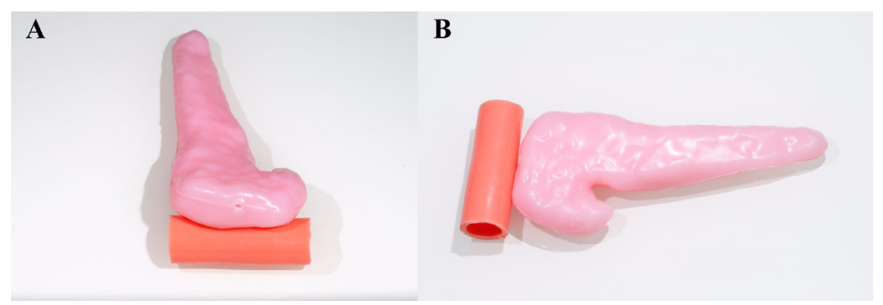

Figure 1 The appearance of the 3D-printed PJ model. (A) The 3D-printed PJ model is primarily composed of three parts: the pancreatic parenchyma, the pancreatic duct and the intestinal duct. (B) Side view of the 3D-printed PJ model. 3D, three dimensional; PJ, pancreaticojejunostomy. with a two-dimensional shear-wave elastography (2D$\mathrm{SWE}$ ) value of nine times.

\section{Patient and public involvement}

Neither patients nor the public were directly involved in the design of the current study.

\section{Evaluation scale design}

The expert evaluation scale of the model was comprehensively designed with reference to the relevant literature, ${ }^{10-12}$ using a 5-point Likert scale (see online supplemental appendix S2). The main coverage areas include the amount of pancreatic surgery the expert had conducted, the evaluation of the overall settings of the 3D-printed model, the evaluation of the appearance, size and tactile similarity of the 3D-printed model and a comprehensive evaluation of the 3D-printed pancreas model for clinical and teaching work.

The model's operation rating scale was designed with reference to the relevant model training literature, ${ }^{13}$ which primarily evaluates the depth perception, force/ tissue handling, dexterity, coordination of the arms and the efficiency of the chief surgeon (attending), first assistant (fellow), and observer (resident) physicians in pancreatic surgery.

The functional psychology scale of the model refers to the NASA Task Load Index (NASA-TLX), which primarily evaluates the mental load of pancreatic surgeons. The significance of the related indices is reported in several articles as it relates to surgical model training. ${ }^{145}$

\section{Assessment scale issuance}

The current study selected eight pancreatic surgery experts and sent the 3D-printed pancreas models and distributed the 3D-printed pancreas model evaluation scales to each of the experts. Experts in pancreatic surgery were invited to participate in the evaluation from all aspects according to the scale and to make professional recommendations.

Fifteen chief surgeons (attendings), first assistants (fellows) and observers (residents) from the general surgery department were selected and issued basic information collection forms. All surgeons in the section provided written informed human participant consent. Model training operations were performed after teaching the procedures. The entirety of the operation was recorded on video and the proficiency was scored by two pancreatic experts who were blinded to the identities of surgeons. After the operation, all personnel were issued a NASA-TLX scale to assess the mental load of the operation.

\section{General information of pancreatic surgeons}

Five attendings, including two experts from the PJ anatomical evaluation department, five fellows and five residents were invited to participate in the current study (general information of the physicians is shown in table 1). There were significant differences in the working years of the three groups of surgeons $(13.40 \pm 3.21$ vs $6.00 \pm 1.22$ vs $2.60 \pm 1.82$, respectively; $\mathrm{p}<0.001)$, in which 
Table 1 General information of attendings, fellows and residents

\begin{tabular}{|c|c|c|c|c|}
\hline & Attendings $(n=5)$ & Fellows $(n=5)$ & Residents $(n=5)$ & P-value \\
\hline Years of working & $13.40 \pm 3.21$ & $6.00 \pm 1.22$ & $2.60 \pm 1.82$ & $<0.001^{\star \star *}$ \\
\hline Cases of pancreatoenterostomy as lead surgeon & & & & $0.008^{\star *}$ \\
\hline$<10$ & $1 / 5(20 \%)$ & $1 / 5(20 \%)$ & $0 / 5(0 \%)$ & \\
\hline$\geq 10$ & $4 / 5(80 \%)$ & $0 / 5(0 \%)$ & $0 / 5(0 \%)$ & \\
\hline 0 & $0 / 5(0 \%)$ & $0 / 5(0 \%)$ & $2 / 5(40 \%)$ & \\
\hline$<10$ & $0 / 5(0 \%)$ & $3 / 5(60 \%)$ & $3 / 5(60 \%)$ & \\
\hline $10-50$ & $0 / 5(0 \%)$ & $1 / 5(20 \%)$ & $0 / 5(0 \%)$ & \\
\hline$>50$ & $5 / 5(100 \%)$ & $1 / 5(20 \%)$ & $0 / 5(0 \%)$ & \\
\hline Number of right handers & $5 / 5(100 \%)$ & $5 / 5(100 \%)$ & $5 / 5(100 \%)$ & 1.000 \\
\hline
\end{tabular}

${ }^{*} p<0.05,{ }^{* *} p<0.01,{ }^{* * *} p<0.001$.

VR, virtual reality.

all attendings had worked for more than 8 years and all residents had worked for 5 or fewer years. The three groups of surgeons had a statistically significant difference both in the number of cases of pancreatoenterostomies as lead surgeons $(p=0.008)$ as well as in the number of cases of pancreaticoduodenectomy as the first assistant $(p=0.014)$. All pancreatic surgeons who participated in the study were right handed, with no significant statistical difference between the three groups of surgeons in simulation training $(p=0.287)$, nor were there any significant statistical differences between the three groups of participants in Virtual Reality surgical training $(\mathrm{p}=0.562)$.

\section{Operation procedures}

The operation procedures used in the current study refer to the classic Cattell-Warren anastomosis method. The operation steps are detailed in figure 2.

\section{Data analyses}

The current study collected statistics on the overall settings and appearance, size and tactile similarity of the 3D-printed pancreas model and the functional evaluation indicators of the model (primarily including the surgical operation score, operation time and NASA-TLX score). Microsoft Excel (V.2016) was used to establish the scoring and evaluation table of each item in the evaluation scale by experts. SPSS (V.20.0, SPSS, Chicago, Illinois) software was then used for the subsequent data analyses and processing. All tests were two tailed and $\mathrm{p}<0.05$ was considered statistically significant. The results from the statistical analyses were entered into Graphpad Prism V.7.0 and related charts were drawn. Each score was calculated by the mean $\pm \mathrm{SD}$.

\section{RESULTS}

Pancreatic surgery experts' anatomical evaluation of the model

The research invited eight pancreatic surgery experts to conduct a comprehensive evaluation. All experts had
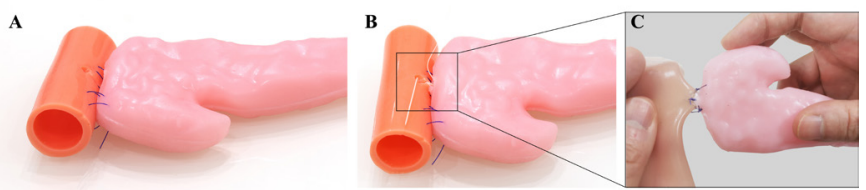

D
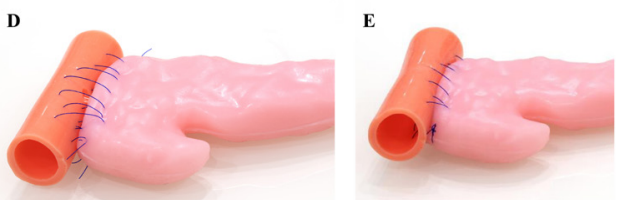

Figure 2 Cattell-Warren anastomosis instructions (A) continuously suture the posterior margin of the pancreas and the seromuscular layer of the jejunum; $2 / 3$ of the pancreatic tissue on the dorsal side of the pancreas should be sutured. The sutures should not be temporarily tightened to facilitate exposure of the posterior pancreatic duct wall. (B) Cut the full thickness of the jejunum wall corresponding to the position of the pancreatic duct. When suturing the posterior wall of the pancreatic duct, $1 / 3$ of the surrounding pancreatic tissue should be included, then knot it together. The knot should be on the outside of the anastomosis. (C) Suture the pancreatic duct and the intestinal duct intermittently at 3, 6, 9 and 12 o'clock, respectively, to complete the anastomosis of the pancreatic duct and the jejunum wall. (D) The anterior wall of the pancreatic duct and its surrounding $1 / 3$ of the pancreatic tissue and the entire anterior wall of the jejunum should be continuously sutured with the suture that was used when the posterior wall was sutured. (E) Tighten the sutures to complete the anastomosis. 

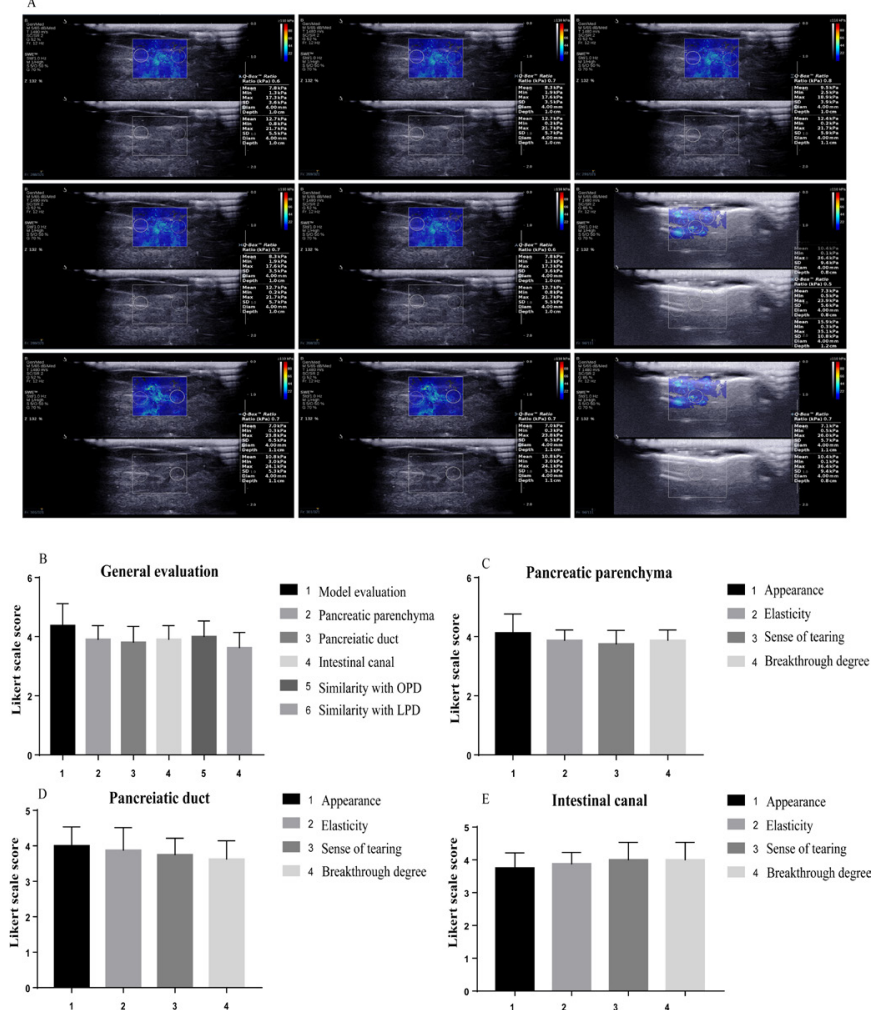

Figure 3 (A) The pancreas stiffness of the PJ model was measured by ultrasound with a 2D-SWE value of $10.08 \pm 6.50$ $\mathrm{kPa}$. (B) General evaluation of the model. (C, D and E) The appearance, elasticity, sense of tearing and breakthrough degree of evaluation of the various parts of the model, including the pancreatic parenchyma, pancreatic duct, and the intestinal canal. 2D-SWE, two-dimensional shear-wave elastography; LPD, laparoscopic pancreaticoduodenectom; OPD, open pancreaticoduodenectomy.

performed more than 20 cases of pancreaticoduodenectomy within the prior year and four had performed more than 100 cases of pancreaticoduodenectomy in the prior year. The model obtained an overall evaluation of $4.38 \pm 0.74$ (figure $3 \mathrm{~B}-\mathrm{E}$ ) and all experts gave greater than 'more similar' (three points) as their evaluation. The current study also invited experts to make assessments on their recommendation of using this model for teaching. The results are presented below.

\section{Appearance}

The overall appearance of the 3D-printed PJ dry laboratory model was evaluated at $3.96 \pm 0.55$. The appearance of the pancreatic parenchyma was evaluated at $4.13 \pm 0.64$, the appearance of the pancreatic duct was evaluated at $4.00 \pm 0.53$ and the appearance of the intestinal canal was evaluated at $3.75 \pm 0.46$.

\section{Tactile sensations}

The pancreas was the primary component of the PJ model and its stiffness was measured via ultrasound with a 2D-SWE value of $10.08 \pm 6.50 \mathrm{kPa}$ (figure $3 \mathrm{~A}$ ). The stiffness of the PJ model was slightly higher $(\mathrm{p}=0.003)$ than that of human tissue, which has been reported as $7.72 \pm 2.50 \mathrm{kPa}^{16}$
The overall tactile evaluation of the 3D-printed PJ dry laboratory model by experts was evaluated at $3.85 \pm 0.46$. The elasticity of the model was evaluated at $3.88 \pm 0.45$ and the elasticity of the pancreas parenchyma, pancreatic duct and intestinal duct of the model were equivalent. The ease of tearing of the model was evaluated at $3.83 \pm 0.48$ and the ease of tearing of the intestinal duct of the model was slightly higher than the other two parts, at $4.00 \pm 0.53$. The suture breakthrough of the model was evaluated at $3.83 \pm 0.48$ and the pancreatic parenchyma of the model was slightly lower than the other two, at $3.88 \pm 0.35$.

\section{Education}

All eight experts (100\%) agreed that the 3D-printed laboratory model of the PJ could/should be used for teaching.

\section{Model functional evaluation}

The functional evaluation of the 3D-printed PJ dry laboratory model included three outcome indicators selected for evaluation, including operation time, operation score and the NASA-TLX score. Details are shown in tables 2 and 3 .

\section{Operation time}

There were significant statistical differences in the operation time of the three groups of researchers $(\mathrm{p}=0.003)$ (shown in figure 4A), where the operation time of the resident group was significantly longer than either that of the fellow group $(1254.80 \pm 341.50$ vs $797.80 \pm 186.40$, $\mathrm{p}=0.028)$ or the attending group $(1254.80 \pm 341.50$ vs $569.20 \pm 170.01 ; \mathrm{p}=0.009)$, but there was no significant statistical difference between the attending group and the fellow group $(569.20 \pm 170.01$ vs $797.80 \pm 186.40, \mathrm{p}=0.175)$.

\section{Operation score}

The operation score for the three groups of researchers was statistically significant $(\mathrm{p}<0.001)$, as shown in figure $4 \mathrm{~B}$, where the operation score of the attending group is significantly higher than fellow group $(18.80 \pm 0.84$ vs $17.20 \pm 0.84, \mathrm{p}=0.023)$ and the resident group $(18.80 \pm 0.84$ vs $14.40 \pm 1.34, \mathrm{p}=0.008)$.

\section{NASA-TLX score}

The NASA-TLX mental load scores of the three groups of researchers were statistically significantly different $(\mathrm{p}=0.031)$, as shown in figure 4C. The NASA-TLX score of the attending group was not significantly different from that of the fellow group (265.40 \pm 99.02 vs $261.60 \pm 86.41$, $\mathrm{p}=0.754$ ), while the NASA-TLX score of the resident group was significantly higher than fellow group $(412.80 \pm 79.74$ vs $261.60 \pm 86.41, \mathrm{p}=0.028)$ and the attending group (412.80 \pm 79.74 vs $265.40 \pm 99.02, \mathrm{p}=0.047$ ).

\section{DISCUSSION}

Traditional surgical teaching and training methods are experiencing increasing learning costs under the modern background and pancreatic surgery is known for its relatively higher surgical difficulty. Within the digestive 
Table 2 The operation time, operation score and the NASA-TLX score of the three groups

\begin{tabular}{|c|c|c|c|c|}
\hline & Attendings $(n=5)$ & Fellows $(n=5)$ & Residents ( $n=5)$ & P-value \\
\hline Operation time & $569.20 \pm 170.01$ & $797.80 \pm 186.40$ & $1254.80 \pm 341.50$ & $0.003^{\star \star}$ \\
\hline Operation score & $18.80 \pm 0.84$ & $17.20 \pm 0.84$ & $14.40 \pm 1.34$ & $<0.001^{\star \star *}$ \\
\hline
\end{tabular}

${ }^{*} \mathrm{p}<0.05,{ }^{* *} \mathrm{p}<0.01,{ }^{* * *} \mathrm{p}<0.001$.

NASA-TLX, NASA Task Load Index.

tract anastomosis, the $\mathrm{PJ}$ is the most complicated, which can lead to various postoperative complications. The PJ model based on biotissue ${ }^{11}$ is considered to improve technical performance in surgical oncology fellows. However, to our knowledge, although they have been successfully applied to training in many fields of surgery, including head and neck surgery, ${ }^{17}$ colorectal surgery, ${ }^{18}$ vascular surgery $^{19}$ and neurosurgery, ${ }^{20}$ among others, there are few reports on PJ models using 3D-printed models.

Elastography is an ultrasound imaging method that has been used to assess the stiffness of tissues. The concept of elastography was first proposed in $1991 .^{21}$ During an elastography evaluation, the stiffness of the model can be estimated from the response of the model to compression. This process can be performed in two ways; shear wave elastography (SWE) or strain elastography. ${ }^{22}$ The current study used soft silicone material to simulate the pancreatic parenchyma and its hardness, which was slightly higher than that of the pancreatic tissue. Our team has also studied hydrogel as a $3 \mathrm{D}$ printing material to print PJ models. Its hardness is very close to that of the pancreas. But the moisture in hydrogel tends to evaporate over time, which causes difficulties with storage, thereby limiting its use. Future studies are planned to conduct in-depth research on this softer material. In the current study, eight pancreatic surgery experts were selected, all of whom exceeded the experience expectations for a pancreaticoduodenectomy, and a model evaluation scale was issued to these experts. The evaluation scale adopts the 5-point Likert scale, ${ }^{10-12}$ which comprehensively evaluates the appearance and touch of each component of the model, its similarity with real surgery and its application in teaching. Experts rated the model highly on both appearance and touch, suggesting that the model has good simulation performance. All experts recommend it

Table 3 P-value of the pairwise group comparison

\begin{tabular}{llll}
\hline & A vs F & A vs R & F vs R \\
\hline Operation time & 0.175 & $0.009^{\star *}$ & $0.028^{\star}$ \\
Operation score & $0.023^{\star}$ & $0.008^{\star *}$ & 0.09 \\
NASA-TLX score & 0.754 & $0.047^{\star}$ & $0.028^{\star}$ \\
\hline
\end{tabular}

${ }^{*} \mathrm{p}<0.05,{ }^{* *} \mathrm{p}<0.01,{ }^{* * *} \mathrm{p}<0.001$.

A, attending group; F, fellow group; NASA-TLX, NASA Task Load Index; R, resident group. for teaching, suggesting a potential role of such models in surgical training.

The current study also selected three groups of surgeons to perform functional tests of the model. The selected research indicators primarily include operation time, operation score and the NASA-TLX. There is a plethora of research on operation time and operation score, which can effectively reflect the operation level on the model. ${ }^{23} 24$ Additionally, Beard et $a l^{25}$ developed an objective-structured assessment of technical skills (OSATS) scale based on the surgeon's technical competency evaluation. The research published by Wei et al ${ }^{13}$ was optimised on the basis of OSATS and was demonstrated to be a good assessment of the technical competency of surgeons. The operation scoring standard of the current research also refers to this modified version of the scoring design. In addition, the current study used the NASA-TLX as a subjective index to assess mental workload, which can reflect the surgeon's operating pressure, which has attracted increasing attention in recent years. ${ }^{1426}$ Given the results of the above three indicators, the model is suggested to be able to effectively distinguish between the three groups of physicians in terms of operating time, operating scores and mental stress, further indicating the effectiveness of the model. Among the groups, the attending group had a shorter operating time than the fellow group (569.20 \pm 170.01 vs $797.80 \pm 186.40)$; however, this difference was not statistically significant.

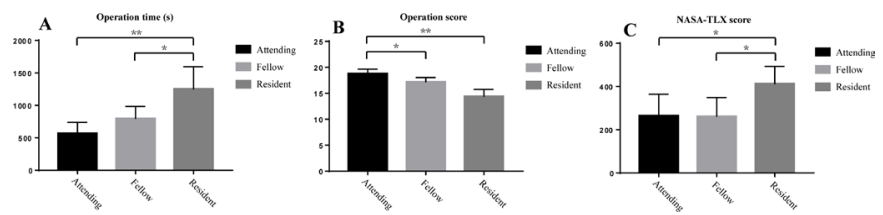

Figure 4 (A) The operation time of the resident group was significantly longer than either that of the fellow group (1254.80 \pm 341.50 vs $797.80 \pm 186.40, p=0.028)$ or the attending group $(1254.80 \pm 341.50$ vs $569.20 \pm 170.01$, $\mathrm{p}=0.009$ ); (B) the operation score of the attending group was significantly higher than either that of the fellow group (18.80 \pm 0.84 vs $17.20 \pm 0.84, p=0.023)$ or the resident group (18.80 \pm 0.84 vs $14.40 \pm 1.34, p=0.008)$; $(C)$ the NASA-TLX score of the resident group was significantly higher than either that of the fellow group $(412.80 \pm 79.74$ vs $261.60 \pm 86.41, p=0.028$ ) or the attending group (412.80 \pm 79.74 vs $265.40 \pm 99.02, p=0.047) .{ }^{*} p<0.05,{ }^{* *} p<0.01$, ${ }^{\star * \star} \mathrm{p}<0.001$. 
This may be due to an insufficient number of enrolled physicians. Additionally, there was no significant difference between the attending group and the fellow group doctors in terms of stress scores, plausibly due to a better psychological tolerance in the fellow group as the amount of surgery gradually increased. Furthermore, the mental stress of attendings and fellows in the model training were significantly lower than that of the residents, suggesting that the model can effectively simulate mental stress. The results of the current study demonstrate that the 3D-printed PJ model has good simulation and effectiveness. It can help distinguish pancreatic surgeons at various levels, which can roughly assess whether pancreatic surgeons are prepared for surgery.

Organ models cut from cadaver tissue have certain advantages in training young doctors in the fields of trauma, plastic surgery, gynaecology, general surgery and vascular surgery. For example, SIM Life, which is an emerging model that uses corpses as a template to have an artificial heartbeat, circulation and breathing, has been given high ratings by users. However, the application of living tissues has many problems such as storage, production and cost. The cost of 3D-printed organisational models is greatly reduced and due to advances in technology and materials, it has improved organisational similarity and training effects, and it is easier to promote and train economically. Simultaneously, it is easier to produce with a short production cycle and it has a better prospect in clinical application.

However, the current study has some disadvantages. One of the limitations that future research should consider is the printing of the pancreas model with the inclusion of vessels, such as the splenic artery, as this will allow for the simulation of a more realistic situation. Additionally, characteristics of the pancreatic tissue (consistency, elasticity, etc) are highly different from one patient to another and influence both the technique and the results of the pancreatoenteric anastomosis. In the current study, only one type of silicon model was used. Furthermore, while a soft silicone material was selected to simulate the pancreatic parenchyma, its hardness was still slightly higher than that of the pancreatic tissue. Additionally, while we chose 15 surgeons performed a PJ on the three-dimensional model, the sample size could be larger. In future studies, different materials should be tried to achieve better material simulation and compare their different training effects and expert evaluation. We also selected the open pancreaticoduodenal model for training and will use the laparoscopic model for additional future research.

\section{CONCLUSIONS}

The three-dimensional PJ model could mimic real surgical situations and can be used to distinguish surgeons of various levels of experience. Therefore, prior to doing a pancreaticoduodenectomy, this model may be a convenient tool to let surgeons to evaluate whether they are technically proficient to perform a high-quality and safe PJ on their patients.

Author affiliations

${ }^{1}$ Key Laboratory of Laparoscopic Technology of Zhejiang Province, Zhejiang University School of Medicine, Sir Run Run Shaw Hospital, Hangzhou, Zhejiang,

China

${ }^{2}$ Department of Thoracic Surgery, Zhejiang University School of Medicine, Sir Run Run Shaw Hospital, Hangzhou, Zhejiang, China

${ }^{3}$ Key Laboratory of Laparoscopic Technology of Zhejiang Province, Zhejiang University School of Medicine, Sir Run Run Shaw Hospital, Hangzhou, China ${ }^{4}$ Department of General Surgery, Zhejiang University School of Medicine, Sir Run Run Shaw Hospital, Hangzhou, Zhejiang, China

${ }^{5}$ Department of General Surgery, Wenzhou Hospital of Integrated Traditional Chinese and Western Medicine, Wenzhou, China

${ }^{6}$ Department of Hepatobiliary and Pancreatic Surgery, Hangzhou Medical College, Hangzhou, China

${ }^{7}$ Department of Research and Development, Ningbo Trandomed 3D Medical Technology Co., Ltd, Ningbo, Zhejiang, China

${ }^{8}$ Department of Oncology, Hangzhou Medical College, Hangzhou, China

${ }^{9}$ Department of Hepatobiliary and Pancreatic Surgery, The First People's Hospital of Tongxiang City, Jiaxing, Zhejiang, China

Correction notice This article has been corrected since it first published. 'Zhifei Wang' has been added as the corresponding author.

Twitter Hao Yu @HaoYu19261955

Acknowledgements The authors would first and foremost like to thank all of the surgeons who participated in the current trial. Furthermore, the authors would like to thank Dr. Jin Yang, Prof. Zhifei Wang, Dr. Tunan Yu and Jiulong Wang for planning the research and Dr. Fangqiang Wei, Haibo Gong, Jiulong Wang and Xinzhong He for conducting the research. We would also like to thank Dr. Haiying Dong, Zhifei Wang, Jin Yang and Tunan Yu for the reporting of the work. The authors would additionally like to thank all the colleagues at Sir Run Run Shaw Hospital who contributed to this research.

Contributors HY, JY, ZW, TY, JW co-conceived the study design, planned and prepared study protocols. HY, FW, HG, JW, XH conducted the research including data collection, data analysis and data interpretation. HY, HD, ZW, JY and TY reported the work. JY and ZW is responsible for the overall content as guarantor.

Funding The current work was supported by the funding of the subproject of the Key R\&D Program of the Ministry of Science and Technology (2018YFB1107104); the Education Reform Project of Zhejiang University School of Medicine (zgyb20202027); the 2015 Natural Science Foundation of Zhejiang Province (Grant Number Y15H160162); the Public Welfare Technology Research Programme/ Social Development of Zhejiang Natural Science Foundation Committee (Number LGF21H030011); the fund of Zhejiang Medical and Health Science and Technology Project (Number 2021KY027); and the fund of 2018 Zhejiang Charity Project (Number Lgf18h030004).

Competing interests None declared.

Patient consent for publication Not applicable.

Ethics approval The Sir Run Run Shaw Hospital granted ethical approval to conduct the current study within its facilities (Ethical Application Ref: jm420-c5a3d, see appendix S2). All procedures followed were in accordance with the ethical standards of the responsible committee on human experimentation (institutional and national) and with the Helsinki Declaration of 1975, as revised in 2000 (5).

Provenance and peer review Not commissioned; externally peer reviewed.

Data availability statement Data are available in a public, open access repository. All data that used in the writing of our article in the text are publicly available and the reference list were cited. See Doi: https://doi.org/10.5281/zenodo.5842799.

Supplemental material This content has been supplied by the author(s). It has not been vetted by BMJ Publishing Group Limited (BMJ) and may not have been peer-reviewed. Any opinions or recommendations discussed are solely those of the author(s) and are not endorsed by BMJ. BMJ disclaims all liability and responsibility arising from any reliance placed on the content. Where the content includes any translated material, BMJ does not warrant the accuracy and reliability of the translations (including but not limited to local regulations, clinical guidelines, terminology, drug names and drug dosages), and is not responsible 
for any error and/or omissions arising from translation and adaptation or otherwise.

Open access This is an open access article distributed in accordance with the Creative Commons Attribution Non Commercial (CC BY-NC 4.0) license, which permits others to distribute, remix, adapt, build upon this work non-commercially, and license their derivative works on different terms, provided the original work is properly cited, appropriate credit is given, any changes made indicated, and the use is non-commercial. See: http://creativecommons.org/licenses/by-nc/4.0/.

\section{ORCID iD}

Hao Yu http://orcid.org/0000-0002-0219-1053

\section{REFERENCES}

1 Ecker BL, McMillan MT, Asbun HJ, et al. Characterization and optimal management of high-risk pancreatic anastomoses during pancreatoduodenectomy. Ann Surg 2018;267:608-16.

2 Besselink MG, van Rijssen LB, Bassi C, et al. Definition and classification of chyle leak after pancreatic operation: a consensus statement by the International Study Group on pancreatic surgery. Surgery 2017;161:365-72.

3 Suzuki S, Kajiyama H, Takemura A, et al. The clinical outcomes after total pancreatectomy. Dig Surg 2017;34:142-50.

4 Szasz P, Louridas M, Harris KA, et al. Assessing technical competence in surgical trainees: a systematic review. Ann Surg 2015;261:1046-55.

5 Ziv A, Wolpe PR, Small SD, et al. Simulation-Based medical education: an ethical imperative. Acad Med 2003;78:783-8.

6 Frank JR, Snell LS, Cate OT, et al. Competency-Based medical education: theory to practice. Med Teach 2010;32:638-45.

7 Tack P, Victor J, Gemmel P, et al. 3D-printing techniques in a medical setting: a systematic literature review. Biomed Eng Online 2016;15:115.

8 Perica ER, Sun Z. A systematic review of three-dimensional printing in liver disease. J Digit Imaging 2018;31:692-701.

9 Sun Z, Lee SY. A systematic review of 3-D printing in cardiovascular and cerebrovascular diseases. Anatol J Cardiol 2017;17:423-35.

10 Kiely DJ, Gotlieb WH, Jardon K, et al. Advancing surgical simulation in gynecologic oncology: robotic dissection of a novel pelvic lymphadenectomy model. Simul Healthc 2015;10:38-42.

11 Tam V, Zenati M, Novak S, et al. Robotic pancreatoduodenectomy Biotissue curriculum has validity and improves technical performance for surgical oncology fellows. J Surg Educ 2017;74:1057-65.
12 Maricic MA, Bailez MM, Rodriguez SP. Validation of an inanimate low cost model for training minimal invasive surgery (mis) of esophageal atresia with tracheoesophageal fistula (AE/TEF) repair. J Pediatr Surg 2016;51:1429-35.

13 Wei F, Xu M, Lai X, et al. Three-Dimensional printed dry lab training models to simulate robotic-assisted pancreaticojejunostomy. ANZ J Surg 2019;89:1631-5.

14 Lowndes BR, Forsyth KL, Blocker RC, et al. NASA-TLX assessment of surgeon workload variation across specialties. Ann Surg 2020;271:686-92.

15 Law KE, Lowndes BR, Kelley SR, et al. NASA-Task load index differentiates surgical approach: opportunities for improvement in colon and rectal surgery. Ann Surg 2020;271:906-12.

16 Sezgin O, Yaraș S, Özdoğan O. The course and prognostic value of increased pancreas stiffness detected by ultrasound elastography during acute pancreatitis. Pancreatology 2021;21:1285-90.

17 Werz SM, Zeichner SJ, Berg B-I, et al. 3D printed surgical simulation models as educational tool by maxillofacial surgeons. Eur J Dent Educ 2018;22:e500-5.

18 Bangeas P, Drevelegas K, Agorastou C, et al. Three-Dimensional printing as an educational tool in colorectal surgery. Front Biosci 2019;11:29-37

19 Wang C, Zhang L, Qin T, et al. 3D printing in adult cardiovascular surgery and interventions: a systematic review. J Thorac Dis 2020;12:3227-37.

20 Weinstock P, Rehder R, Prabhu SP, et al. Creation of a novel simulator for minimally invasive neurosurgery: fusion of 3D printing and special effects. J Neurosurg Pediatr 2017;20:1-9.

21 Ophir J, Céspedes I, Ponnekanti H, et al. Elastography: a quantitative method for imaging the elasticity of biological tissues. Ultrason Imaging 1991;13:111-34.

22 Kuwahara T, Hirooka Y, Kawashima H, et al. Quantitative evaluation of pancreatic tumor fibrosis using shear wave elastography. Pancreatology 2016;16:1063-8.

23 Pucci JU, Christophe BR, Sisti JA, et al. Three-Dimensional printing: technologies, applications, and limitations in neurosurgery. Biotechnol Adv 2017;35:521-9.

24 Bartel T, Rivard A, Jimenez A, et al. Medical three-dimensional printing opens up new opportunities in cardiology and cardiac surgery. Eur Heart J 2018;39:1246-54.

25 Beard JD, Education and Training Committee of the Vascular Society of Great Britain and Ireland. Setting standards for the assessment of operative competence. Eur J Vasc Endovasc Surg 2005;30:215-8.

26 Abbott EF, Thompson W, Pandian TK, et al. Personalized video feedback and repeated task practice improve laparoscopic KnotTying skills: two controlled trials. Acad Med 2017;92:S26-32. 